\title{
The case for integrated pediatric behavioral health care: translating research into policy and practice
}

\author{
Nicole Wilberding and Robert D Friedberg* \\ Center for the Study and Treatment of Anxious Youth at Palo Alto University, USA
}

Pediatric primary care practices represent the front-lines for treating young patients' behavioral health problems. Nearly half of all pediatric primary visits include a behavioral health concern [1]. More specifically, behavioral health complaints account for the five chief reasons for seeing a pediatrician [2]. Although approximately 1 in 5 children have a diagnosable mental health condition, less than half of children and parents needing mental health services receive treatment [2-4]. Recent studies indicate that although pediatric behavior health concerns present frequently, they regularly remain undiagnosed and are commonly untreated [3]. Alarmingly, emergency room visits due to behavioral health problems increased 47 per cent over a ten year period from 2003-2012 and 1 in 10 pediatric hospitalizations are related to a psychiatric reason boosting health care costs [2]. Some researchers [5] hypothesize that reductions in inpatient psychiatric beds and a scarcity of skilled clinicians propel this rise in ER visits. Further, across racial and ethnic groups, the leading children's health challenges that worry parents are all behavioral problems [6]. In this brief commentary, we make the case for integrated pediatric behavioral health care based on the research examining its use.

The American Academy of Pediatrics' (AAP) encourages integrated pediatric primary behavioral health care. Indeed, the number of integrated practices are surging [4,7]. In a recent study [7], pediatric residents nearly unanimously agreed that pediatricians should be able to diagnose a variety of conditions including ADHD, depression, and anxiety. However, much smaller numbers thought they were competent to treat these disorders. Commenting on the increase of ER visits due to pediatric behavioral health problems, Rogers and colleagues [5. pg. 490) stated, "Pediatric medical education includes mental health, but may be insufficient given this rising population."

A one stop shopping approach to health care that offers treatment for physical and behavioral health concerns offer multitudinous advantages $[2,4,6,8-10]$. Increased access, improved continuity of care, decreased stigma, enhance accountability, and potential cost effectiveness/cost containment are some propitious outcomes. In a study encompassing seven Children's Community Pediatric Clinics [11], collaborative care was associated with decreased externalizing and internalizing symptoms, better treatment response, and increased symptom remission. Recent research shows that patients and pediatricians are quite satisfied with integrated primary pediatric behavioral health care [2]. Additionally, integrated and collaborative care models performed better than usual care practices [8].

Continuity of care and collaboration are improved in integrated models. Studies show that both training and practice in an integrated pediatric setting increase pediatricians' referrals for behavioral health problems and decrease their perceived workload burden [2,7].
Pediatric residents training in integrated clinics assessed for ADHD more regularly and were three times more likely to refer patients with depression or behavior problems [7].

Integrated care practices reduce medical expenditures offsetting costs by lowering provider turnover and increasing productivity [4]. Integrated care is cost efficient and effective with approximately a 50 per cent reduction in monthly patient costs [2]. Reduction in costs for patients and improved efficiency as a result of integrated pediatric care are manifested by declining emergency department visits, fewer hospitalizations, and decreased redundancies in care [2].

Changing our health care culture to become more collaborative, accountable, and patient-centered at both the training and practice levels is critical for the success of integrative pediatric care [9]. Practitioners will need to be fluent with brief, efficient, and empirically sound intervention methods [12]. Clearly, efforts at better workforce development is necessary [13]. Pediatricians, psychologists, nurses, and other allied health professionals should be well-trained in interdisciplinary collaboration. Constructing viable payment systems that reimburse providers not only for their clinical intervention time but also for consultation/liaison and primary prevention efforts is crucial [8]. Accordingly, improving health care insurance and financial literacy is essential $[13,14]$. Finally, utilizing practice-based research skills to evaluate clinical outcomes as well as cost effectiveness is imperative.

Current advances in integrative pediatric behavior health care should propel greater implementation. Patients are well-served by more accountable, collaborative, and accessible services. Providers reap benefits of team-based consultation and working in systems that offer multi-levels of care. While certain administrative and financial barriers exist, the considerable promise of integrated pediatric behavioral health care should motivate policy makers and payers to mitigate obstacles.

\section{References}

1. Weitzman CC, Leventhal JM (2006) Screening for behavioral health problems in primary care. Curr Opin Pediatr 18: 641-648. [Crossref]

2. Yogman MW, Betjemann S, Sagaser A, Brecher L (2018) Integrated behavioral health care in pediatric primary care: A quality improvement project. Clin Pediatr 57: 461470. [Crossref]

3. Chaffin M, Campbell C, Whitworth DN, Gillaspy SR, Bard D, et al. (2017) Accuracy of a pediatric screener to detect untreated behavioral health problems in primary care settings. Clin Pediatr 55: 427-434. [Crossref]

${ }^{\star}$ Correspondence to: Robert D Friedberg, Center for the Study and Treatment of Anxious Youth at Palo Alto University, 1791 Arastradero Rd., Palo Alto, USA, E-mail: rfriedberg@paloaltou.edu

Received: March 28, 2018; Accepted: April 16, 2018; Published: April 19, 2018 
4. Stancin T, Perrin EC (2014) Psychologists and pediatricians: Opportunities for collaboration in primary care. Am Psychol 69: 332-343. [Crossref]

5. Rogers SC, Mulvey CH, Divietro S, Sturm J (2017) Escalating mental health care in pediatric emergency departments. Clin Pediatr 56: 488-491. [Crossref]

6. Asarnow, JR Kolko DJ, Miranda J, Kazak AE (2017) The Pediatric Patient-Centered Medical Home: Innovative models for improving behavioral health. Am Psychol 72: 13-27. [Crossref]

7. Ragunanthan B, Frosch EJ, Solomon BS (2017) On-site mental health professionals and pediatric residents in continuity clinic. Clin Pediatr 56: 1219-1226.

8. Asarnow JR, Rozenman M, Wilblin J, Zeltzer L (2015) Integrated medical-behavioral care compared with usual primary care for child and adolescent behavioral health: A meta-analysis. JAMA Pediatr 169: 929-937. [Crossref]

9. Kirch DG, Ast CE (2017) Health care transformation: The role of academic health centers and their psychologists. J Clin Psychol Med Sttng 24: 86-91.
10. Paternostro JK, Rozbruch EV, Friedberg RD (2016) Integrating Psychosocial Interventions with Pediatric Patients with Gastrointestinal Disorders in Primary Care and Specialty Care Services, Internal Medicine Review, in press.

11. Kolko DJ, Campo J, Kilbourne AM, Hart J, Sakolsky D, et al. (2014) Collaborative care outcomes for pediatric behavioral health outcomes: a cluster randomized clinical trial Pediatrics 133: e981-e992. [Crossref]

12. Janicke DM, Fritz AM, Rozensky RM (2015) Healthcare reform and preparing the future clinical child and adolescent psychology workforce. J Clin Child and Adoles Psychol 44: 1030-1039. [Crossref]

13. Serrrano N, Cordes C, Cubic B, Daub S (2017) The state and future of primary care behavioral health model of service delivery workforce. J Clin Psychol Med Sttings, in press. [Crossref]

14. Friedberg RD (2015) Are professional psychology training programs willing the future to economic illiterates?: A clarion call for action. J Mntl Hlth 25: 395-402.

Copyright: $\bigcirc 2018$ Wilberding N. This is an open-access article distributed under the terms of the Creative Commons Attribution License, which permits unrestricted use, distribution, and reproduction in any medium, provided the original author and source are credited. 